\title{
Rare Presentation of an Uncommon Disease
}

\author{
Vikas Marwah, M.D. ${ }^{1}{ }^{(0)}$, Shrinath Vasudevan, M.B.B.S. ${ }^{1}$, Robin Choudhary, \\ M.D. ${ }^{1}$ and Arpitha Pemmaraju, M.D. ${ }^{2}$
}

Departments of ${ }^{1}$ Pulmonary, Critical Care and Sleep Medicine and ${ }^{2}$ Pathology, Army Institute of Cardiothoracic Sciences, Pune, India

Mucormycosis is caused by infection with molds in the order of Mucorales that are ubiquitous in nature. The most common genera associated with mucormycosis are Rhizopus and Mucor. These molds can gain access to the body via inhalation, skin penetration, and ingestion. They can cause a spectrum of illness of the skin and soft tissues, rhino-orbitalcerebral, lower respiratory tract, gastrointestinal, and disseminated infections. The disease is extremely lethal with a high mortality rate for patients with disseminated disease ${ }^{1}$. The disease has recently risen to public fame as "black fungus" complicating the on-going coronavirus disease 2019 pandemic. It affects mostly immunocompromised host ${ }^{2}$. The presence of pulmonary mucormycosis in non-immunocompromised patients is extremely rare ${ }^{3}$. Herein, we report a case of pulmonary mucormycosis in a non-immunocompromised patient who was diagnosed and managed at our center. The written informed consent to publish the case was taken from the Next of Kin. The study protocol was approved by the departmental and institute's committee of medical research.

A 72-year-old female with no known comorbidities presented with complaints of intermittent fever and non-productive cough for one month in duration associated with pleuritic chest pain for one week in duration. She had no history of fever, night sweats, weight loss, wheeze, hemoptysis, or connective tissue disorders. The patient visited the outpatient clinic of another hospital multiple times during the last month wherein she was managed symptomatically with anti-pyretic and oral antibiotics. She had corticosteroids, other immunomodula-

Address for correspondence: Robin Choudhary, M.D.

Department of Pulmonary, Critical Care and Sleep Medicine, Army Institute of Cardiothoracic Sciences, Pune, Maharashtra 411040, India

E-mail: robinch19@gmail.com

Received: Nov. 16, 2021

Revised: Dec. 8, 2021

Accepted: Dec. 11, 2021

Published online: Dec. 13, 2021

(c) It is identical to the Creative Commons Attribution Non-Commercial License (http://creativecommons.org/licenses/by-nc/4.0/). tory agents, surgical interventions, or hospital admission. On examination, her vitals were stable without any evidence of respiratory distress, peripheral stigmata of tuberculosis, or connective tissue disorders. Respiratory examination revealed decreased movement of left hemithorax without signs of volume loss. There were bronchial breath sounds over left infraclavicular and mammary regions.

On evaluation, the patient was anaemic (hemoglobin $9.7 \mathrm{~g} /$ dL, microcytic hypochromic anemia). However, all other hematological and biochemical parameters were within normal ranges. Serological workups for human immunodeficiency virus, hepatitis B virus, and hepatitis $\mathrm{C}$ virus were negative. Her anti-nuclear antibodies, anti-cyclic citrullinated peptide, C-reactive protein, and rheumatoid factor workups were also negative. Chest radiography showed homogenous oval opacity with well-defined borders in the left middle zone (Figure 1A). High resolution computed tomography of the chest showed air space opacities in the left upper lobe's anterior and posterior segments with surrounding ground glass opacities (Figure 1B). Sputum Gram stain, fungal stain, and ZiehlNeelsen stain did not show any microorganisms. Sputum cartridge based nucleic acid amplification test for mycobacterium tuberculosis was negative. Ultrasonographic evaluation of kidney showed normal sized kidneys, normal echotexture, and corticomedullary differentiation with a non-obstructive left renal calculus measuring $5 \mathrm{~mm}$. Video bronchoscopy showed a normal tracheobronchial tree with bronchoalveolar lavage (BAL) negative for bacteria and fungi. BAL fluid cytology showed predominantly alveolar macrophages (55\%) without evidence of any atypical cells. Due to her advanced age, non-resolving pneumonia status, and radiological appearance of lesion, the patient was suspected to have pulmonary malignancy. Thus, computed tomography guided biopsy of the lesion was performed. It revealed broad aseptate hyphae with right angle branching (Figure 1C, D). The patient was diagnosed with pulmonary mucormycosis. She was reviewed by ENT (ear, nose, and throat) specialist. The presence of sinonasal and orbital/ocular mucormycosis mucormycosis was ruled out. Nasal swab potassium hydroxide mount was negative for fungal hyphae.

The patient was counselled regarding the disease and therapeutic options. She was started with liposomal amphotericin 

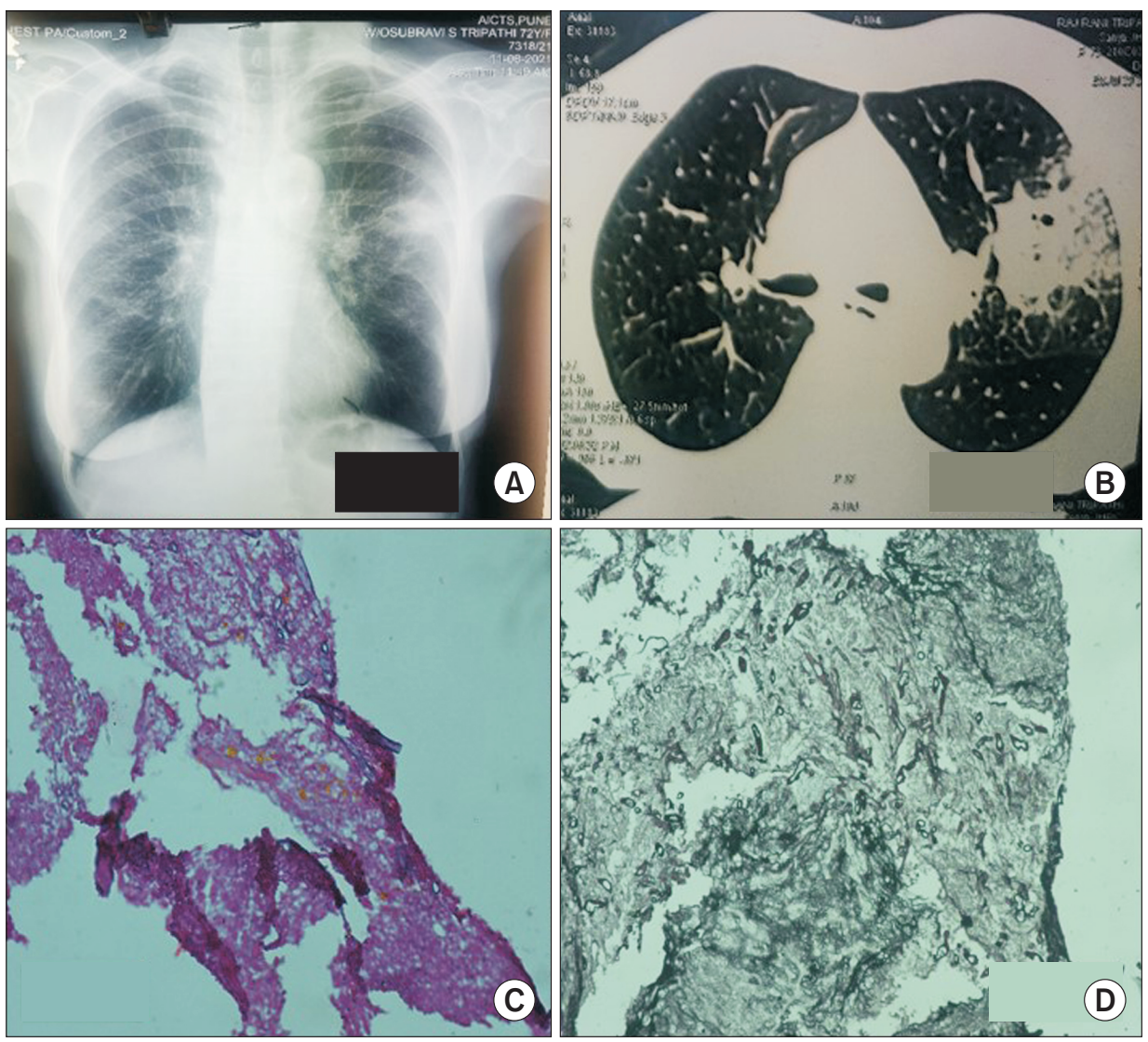

Figure 1. (A) Chest radiograph of the patient showing a homogenous oval opacity with well-defined borders in the left middle zone. (B) High resolution computed tomography of chest showing air space opacities in the left upper lobe's anterior and posterior segments with surrounding ground glass opacities. (C, D) Grocott-Gomori's methenamine silver staining of lung biopsy showing broad, aseptate hyphae with irregular branching $(\mathrm{C}, \times 100 ; \mathrm{D}, \times 400)$.

$\mathrm{B}$ at a dose of $3 \mathrm{mg} / \mathrm{kg}$ which was infused over 2 hours at a concentration of $0.5 \mathrm{mg} / \mathrm{mL}$. On the third day of liposomal amphotericin B therapy, the patient had infusion related adverse effects in the form of fever and chills towards the end of infusion which was managed with intravenous corticosteroids and antihistamines. On day 7 of liposomal amphotericin B therapy, the patient developed acute kidney injury with raised transaminases, hyponatremia, and hypokalaemia due to which the liposomal amphoteric B was with-held. Her condition deteriorated further with the development of upper gastrointestinal bleeding and hyperchloremic normal anion gap metabolic acidosis. She was diagnosed with amphotericin $\mathrm{B}$ induced type IV renal tubular acidosis. She was managed with IV fluids, bicarbonate therapy, proton pump inhibitors, packed red blood cells infusion, and antibiotics. However, she did not respond to the therapy and succumbed to multi-organ dysfunction.

Pulmonary mucormycosis is a sinister infection which is characterized by angioinvasion leading to tissue necrosis and massive hemoptysis. The rapid growth of fungi releases a large number of airborne spores that are constantly inhaled by humans. Even with this constant exposure, infection is rare because an intact immune system can effectively clear these spores. However, an underlying immunocompromising condition makes the host ineffective in clearing these inhaled spores. A meta-analysis of 851 mucormycosis patients by Jeong et al. ${ }^{2}$ has described underlying conditions and predisposing factors for mucormycosis such as diabetes mellitus, hematological malignancies, aplastic anemia, solid organ transplant, hematopoietic stem cell transplant, liver disease, corticosteroid use, neutropenia, major trauma, biological therapy, chemotherapy, calcineurin inhibitors, and renal replacement therapy ${ }^{2}$. The disease is extremely rare in patients without any underlying predisposing factors based on a systematic review of data published between January 1 , 2010 and October 10, 2020 from 15 published articles describing 16 patients ${ }^{3}$. This makes our case report an extremely rare description of a deadly disease. Our patient was extensively evaluated. She had no comorbidities that predisposed her to pulmonary mucormycosis.

Epidemiological review of 929 cases of mucormycosis showed a mortality of $76 \%$ in patients with pulmonary mucormycosis ${ }^{1}$. The mortality was around $43 \%$ in immunocompetent patients with mucormycosis ${ }^{3}$. If left untreated, the disease can have a fatal result. Mucormycosis causing bilateral pulmonary artery aneurysm has also been reported ${ }^{4}$. A combined surgical and medical therapy has mortality benefit over medical management alone. However, as the patient in our study was unwilling to undergo a surgery and immediate treatment initiation could improve survival, patient was started on Inj 
liposomal amphotericin B per recommendation of the European Society for Clinical Microbislogical and Infectious Disease guidelines ${ }^{5}$. The European Confederation of Medical Mycology mucormycosis registry has reported that liposomal amphotericin B at a median dose of $5 \mathrm{mg} / \mathrm{kg} /$ day (range, 3-10 $\mathrm{mg} / \mathrm{kg} /$ day) shows good response rates ${ }^{6}$. Although the recommended dose was $5 \mathrm{mg} / \mathrm{kg} /$ day, the patient was started at a lower dose of $3 \mathrm{mg} / \mathrm{kg} /$ day with a plan to escalate the dose per drug tolerance of patients. The medication was constituted at company recommended concentration of $0.5 \mathrm{mg} / \mathrm{mL}(0.2-2$ $\mathrm{mg} / \mathrm{mL}$ ). It was given using a controlled infusion device over a period of 120 minutes. Despite the best of intentions and careful drug monitoring, the patient did not tolerate the therapy with the development of adverse effects such as drug-induced renal tubular acidosis, liver injury, and upper gastrointestinal bleeding which was found to be fatal.

Pulmonary mucormycosis can rarely affect immunocompetent hosts. Therefore, biopsy taken even from a patient without a predisposing factor should be subjected to fungal staining. The disease is extremely lethal, so is its therapy.

\section{Authors' Contributions}

Conceptualization: Marwah V. Methodology: Marwah V, Choudhary R. Investigation: Marwah V, Vasudevan S, Choudhary R, Pemmaraju A. Writing - original draft preparation: Choudhary R. Approval of final manuscript: all authors.

\section{Conflicts of Interest}

No potential conflict of interest relevant to this article was reported.

\section{Funding}

No funding to declare.

\section{References}

1. Roden MM, Zaoutis TE, Buchanan WL, Knudsen TA, Sarkisova TA, Schaufele RL, et al. Epidemiology and outcome of zygomycosis: a review of 929 reported cases. Clin Infect Dis 2005;41:634-53.

2. Jeong W, Keighley C, Wolfe R, Lee WL, Slavin MA, Kong DCM, et al. The epidemiology and clinical manifestations of mucormycosis: a systematic review and meta-analysis of case reports. Clin Microbiol Infect 2019;25:26-34.

3. He J, Sheng G, Yue H, Zhang F, Zhang HL. Isolated pulmonary mucormycosis in an immunocompetent patient: a case report and systematic review of the literature. BMC Pulm Med 2021;21:138.

4. Marwah V, Tiwari S, Shergill SS, Mishra PS, Bhattacharjee S. Rare case of disseminated mucormycosis presenting with bilateral pulmonary artery aneurysm. Med J DY Patil Vidyapeeth 2021;14:340-2.

5. Cornely OA, Arikan-Akdagli S, Dannaoui E, Groll AH, Lagrou $\mathrm{K}$, Chakrabarti A, et al. ESCMID and ECMM joint clinical guidelines for the diagnosis and management of mucormycosis 2013. Clin Microbiol Infect 2014;20 Suppl 3:5-26.

6. Skiada A, Pagano L, Groll A, Zimmerli S, Dupont B, Lagrou K, et al. Zygomycosis in Europe: analysis of 230 cases accrued by the registry of the European Confederation of Medical Mycology (ECMM) Working Group on Zygomycosis between 2005 and 2007. Clin Microbiol Infect 2011;17:1859-67. 